\title{
Three-Dimensional Structure of Cytoskeletal System in Human Hepatocytes Viewed by Polyethylene-Glycol-Embedding Method
}

\author{
Motoyasu Ishit, Yutaka Miyazaki, Shinichi Ohta, \\ Masao Otsuki and Yoshio Goto \\ Third Department of Internal Medicine, Tohoku University \\ School of Medicine, Sendai 980
}

\begin{abstract}
IshiI, M., Miyazaki, Y., Ohta, S., Otsuki, M. and Goto, Y. ThreeDimensional Structure of Cytoskeletal System in Human Hepatocytes Viewed by Polyethylene-Glycol-Embedding Method. Tohoku J. exp. Med., 1985, 147 (1), 4763 - In an attempt to demonstrate the three-dimensional cytoskeletal system of human hepaocytes, we used two kinds of tissue processing. In one, specimens were immediately fixed with $1 \%$ glutaraldehyde, saponin $0.5 \mathrm{mg} / \mathrm{ml}$, and tannic acid $2 \mathrm{mg} / \mathrm{ml}$ (procedure A) and in the other, cytoplasmic matrix and organelles were washed out with a solution containing saponin $0.5 \mathrm{mg} / \mathrm{ml}$ and $0.15 \%$ Triton $\mathrm{X}-100$, followed by fixation with glutaraldehyde (procedure B). The specimens were embedded in polyethylene glycol, sectioned at $500 \mathrm{~nm}$, critical point-dried and observed in a TEM. With procedure A, a fine lattice was shown to spread throughout the cytoplasm so as to connect plasma membranes with cytoplasmic organelles, condensed around the bile canaliculi. With procedure B, microfilaments, microtubules and intermediate filaments could be clearly visualized three-dimensionally instead of the lattice structure. At a higher magnification, microvilli of canaliculus were found to contain a core microfilament which were enmeshed in a pericanalicular filament plexus. This plexus looking like the terminal web of intestinal epithelia, encircled the canaliculus and was attached to the zonula adherens. Outside the plexus, intermediate filament network was shown attached to the desmosome. These morphological findings indicated that the cytoskeleton of human hepatocytes is composed of microfilaments, microtubules, intermediate filaments and the fine lattice structure, suggesting that the bile canaliculi could contract in vivo. —_ cytoskeleton; human hepatocyte ; polyethylene-glycol-embedding method; three-dimensional electron microscopy
\end{abstract}

Many papers have dealt with the filamentous structures of human hepatocytes and their relation to bile canaliculi (Bieva 1964; Oda et al. 1974; French and Davis 1975; Yokota and Fahimi 1979). The distribution of the filaments can be observed by using immunofluorescence staining (Horoboroco et al. 1975 ; Yokota and Fahimi 1979), but can hardly be seen by conventional electron microscopy because in a two dimensional ultrathin section filamentous structures appear only

Received December 20, 1984. ; accepted for publication June 10, 1985. 
as pin-points. In an electron microscopic study of microfiaments (MFs) in relation to bile canaliculi Oda et al. (1974) showed direct connection of MFs with bile canalicular membrane on isolated bile canaliculi of rat liver. But the exact distribution of the connection remains unclear.

Recently, Wolosewick and Porter $(1976,1979)$ showed a three-dimensional structure of cytoskeletal system, composed of MFs, microtubules (MTs), intermediate filaments (IFs) and microtrabecular lattice in cultured cells by using high voltage electron microscopy. After that, Ellisman and Porter (1980) showed the axoplasmic cytoskeleton in vivo by using polyethylene-glycol (PEG) as a watersoluble embedding material. Applying this method to rat hepatocytes, we noticed that it was necessary for observing the cytoskeleton to wash out cytosolic soluble proteins with detergent solution (Ishii et al. 1984).

The purpose of this study is to observe the cytoskeletal system of human hepatocytes three-dimensionally and to clarify the structural integrity of this system employing a method of fixation and extraction modifying the previously reported one (Ishii et al. 1984).

\section{Materials and Methods}

\section{Patient materials}

Liver specimens used in this study were obtained by needle biopsy from two patients. One was a female, 45 years, having idiopathic portal hypertension and the other, 17 years old male, with clinical diagnosis of non-A, non-B posttransfusion hepatitis. In the first patient, a moderate pancytopenia and splenomegaly were the main clinical features and liver biopsy was done to rule out the presence of chronic liver diseases; the histological diagnosis of the liver was mild portal fibrosis without parenchymal necrosis. Her biochemical data were all within normal range with neither positive HBsAg nor HBsAb. In the second patient, the biochemical data were moderately abnormal, compatible with a healing stage of acute viral hepatitis, but total bilirubin value at the time of liver biopsy was 0.6 $\mathrm{mg} / \mathrm{ml}$ with jaundice never found throughout the course of illness. The histological diagnosis of the liver was non-specific reactive hepatitis. Ultrastructural findings of hepatocytes in either patient were not remarkable.

\section{Procedures for cytoplasmic washout, fixation, embedding and observation}

Extraction and fixation methods were slightly modified but embedding procedure was essentially the same as previously described (Ishii et al. 1984).

Procedure A : A part of biopsy specimen was put into a solution containing $1 \%$ glutaraldehyde, saponin $0.5 \mathrm{mg} / \mathrm{ml}$ and tannic acid $2 \mathrm{mg} / \mathrm{ml}$ in 'stabilization buffer' $(0.5 \mathrm{mM}$ $\mathrm{MgCl}_{2}, 0.1 \mathrm{mM}$ EDTA, 0.1 M PIPES, pH 6.9) (Heuser and Kirschner 1980; Maupin and Pollard 1983), cut into 1 to $3 \mathrm{~mm}^{3}$ and fixed for $45 \mathrm{~min}$ at room temperature. After washing, they were postfixed with $0.1 \% \mathrm{OsO}_{4}$ in phosphate buffer $(\mathrm{pH} 6.4)$ for $20 \mathrm{~min}$ at $4^{\circ} \mathrm{C}$, and then dehydrated in an ethanol series for $10 \mathrm{~min}$ at each step.

Procedure B: The other procedure for extraction and fixation was as follows; small tissue fragments of biopsy sample were incubated in PHEM buffer (60 mM PIPES, $25 \mathrm{mM}$ HEPES, $10 \mathrm{mM} \mathrm{EGTA,} 2 \mathrm{mM} \mathrm{MgCl}$, $\mathrm{pH}$ 6.9) (Schliwa and van Blerkom 1981) containing $0.15 \%$ Triton X-100 and saponin $0.5 \mathrm{mg} / \mathrm{ml}$ for $3 \mathrm{hr}$ at room temperature, followed by fixation with $2.5 \%$ glutaraldehyde in PHEM buffer for $1 \mathrm{hr}$ at room temperature and $1 \%$ $\mathrm{OsO}_{4}$ for $1 \mathrm{hr}$ at $4^{\circ} \mathrm{C}$, and then dehydrated in an ethanol series. 
Some of the specimens treated with either procedure A or B were incubated in an ascending series of $50 \%$ PEG ( $\mathrm{mw}=8,000$, Sigma) in ethanol (vol $/ \mathrm{vol}$ ), $75 \% \mathrm{PEG}$ and $100 \%$ molten $\mathrm{PEG}$ at $80^{\circ} \mathrm{C}$, each for $4 \mathrm{hr}$, transferred to gelatin capsules filled with fresh $100 \%$ molten $\mathrm{PEG}$, and then cooled in a $-20^{\circ} \mathrm{C}$ freezer. Others were embedded in an epon mixture.

Materials embedded in PEG were sectioned by MT-5000 (Sorval) ultramicrotome at a thickness of about $500 \mathrm{~nm}$. Sections were put onto poly-L-lysine-treated formvar-coated grids, dehydrated in ethanol and critical-point-dried with $\mathrm{CO}_{2}$ in a Hiatchi HCP-1 critical point drier. The dried sections were viewed in a Hitachi H-700 electron microscope operated at $200 \mathrm{kV}$, and photographed in a stereopair at a tilting angle of $\pm 10^{\circ}$. Materials embedded in epon mixture were processed for a conventional electron microscopy and viewed in a Hitachi $\mathrm{H}-600$ operated at $75 \mathrm{kV}$.

\section{Immunocytochemistry of actin and keration}

Biopsy samples washed out with procedure B were frozen in OCT compound (Lab-Tek Products, USA) prior to fixation. The samples were washed with phosphate buffer ( $\mathrm{pH} 7.2$ ) and were incubated with 10-fold-diluted anti-actin rabbit IgG or 10-fold-diluted antikeratin rabbit IgG (Transformation Research, Inc. USA) overnight at $4^{\circ} \mathrm{C}$. They were examined with a Nikon fluorescence microscope.

\section{Results}

As described above, we used two schedules of procedure A and B in order to

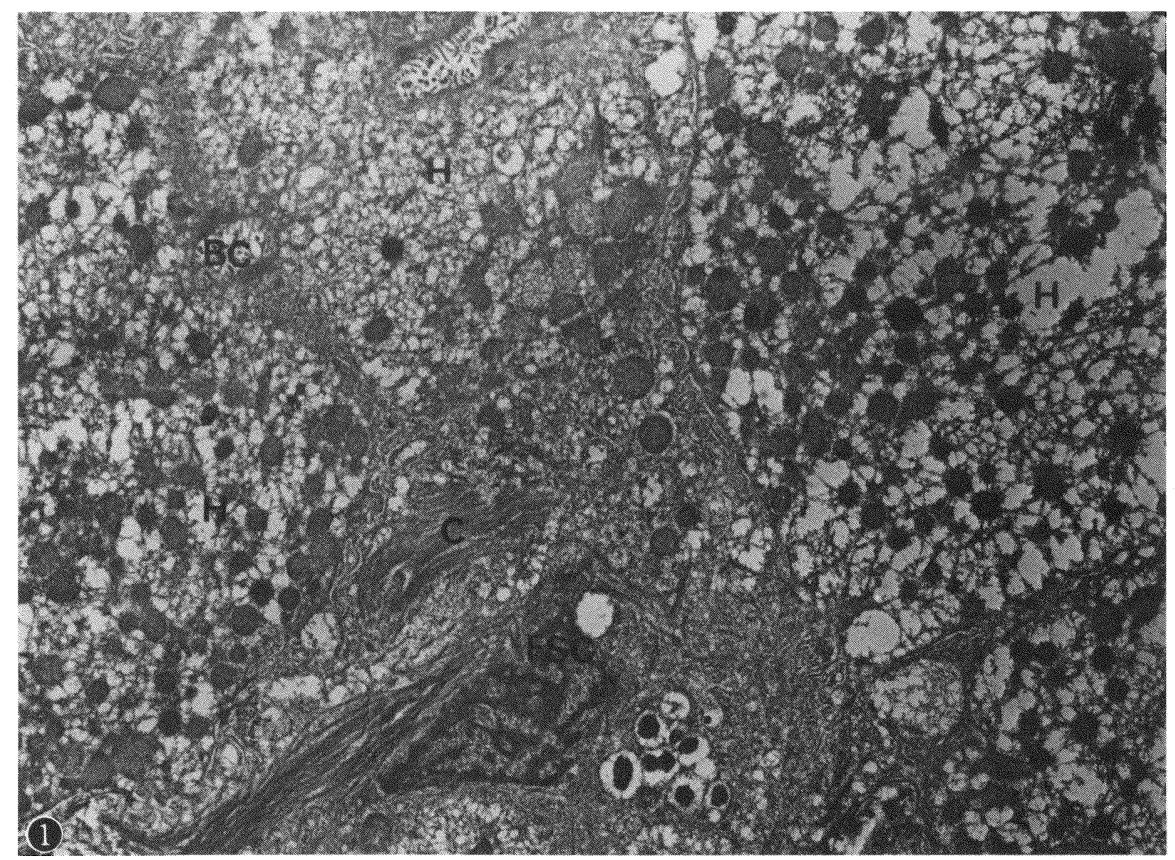

Figs. 1-6. ; Electron micrographs from sections treated with procedure A.

Fig. 1. Low magnification overview of PEG section. Note each constituents of the liver can be seen to preserve the ultrastructure and the intact relationship to each other. $\mathrm{H}$ : hepatocyte FSC: rat-storing cell $\mathrm{C}$ : collagen fibers $\mathrm{BC}$ : bile calaliculus $\times 4,100$ 

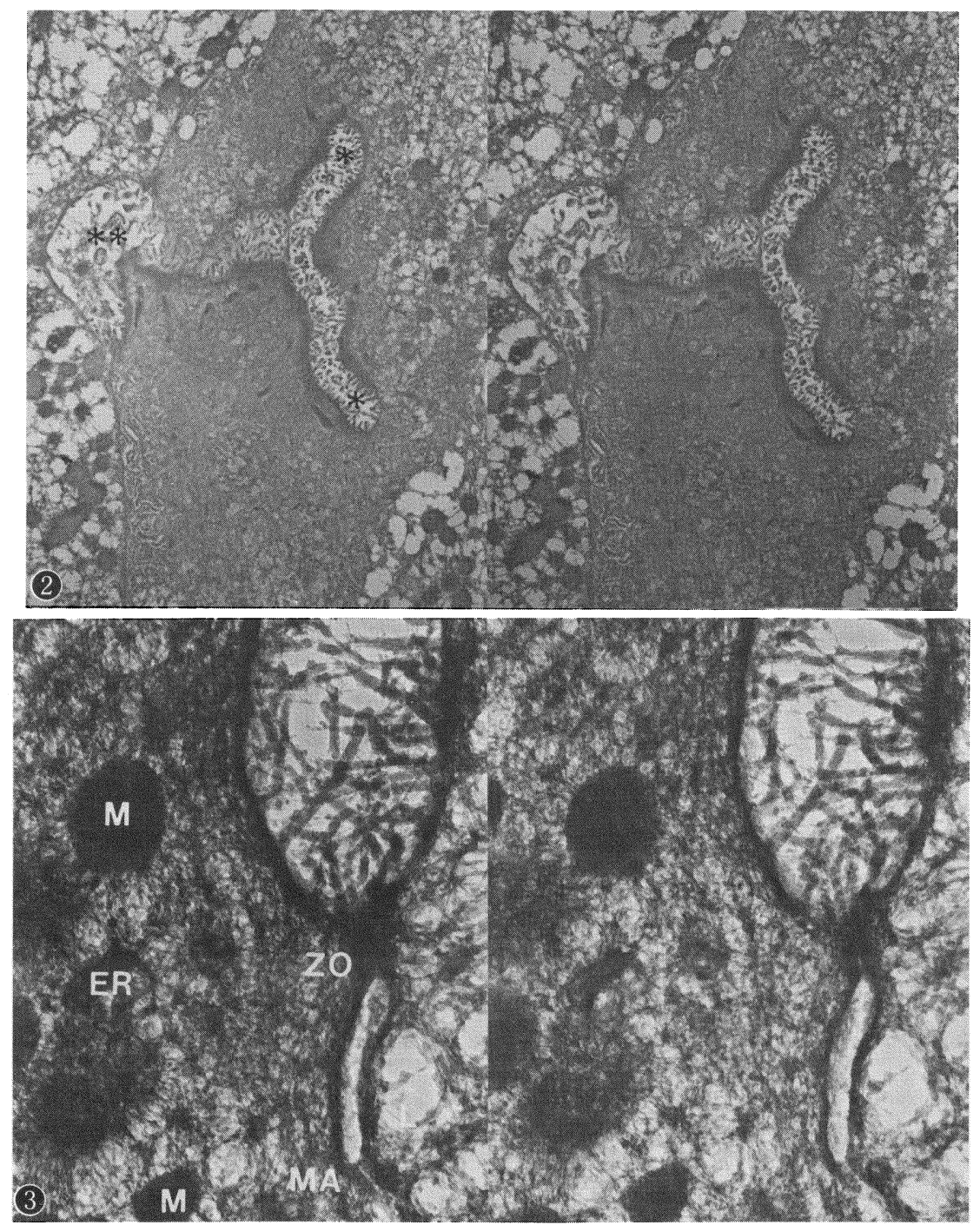

Fig. 2. Stereo pair electron micrographs of two terminal bile canaliculi $(*)$ of longitudinal section. A bile canaliculus running perpendicular to cut surface $(* *)$, around which fine anastomosing filament network was condensely packed. $\times 4,100$

Fig. 3. Stereo pair micrographs of detailed structure of bile canaliculus. Two round organelles interpreted as mitochondria or microbodies (M), and an irregular-shaped one interpreted as endoplasmic reticulum (ER), are embedded within the pericanalicular network. A tight junction (ZO) showing a high density plaque and a desmosome (MA) of less density are also seen. Cross-bridges between plasma membrane and organelles are too condensed that many fibrils are superimposed to make it difficult to discriminate each fibril. $\times 18,000$ 
see different effects of cytoplasmic washout.

\section{Cytoskeletal structure treated with procedure $A$}

Fig. 1 is an electron micrograph from a thick section of liver tissue displaying several hepatocytes and space of Disse at low magnification. Cytoplasmic organelles are mostly lost, but the arrangement of hepatocytes and their attachment

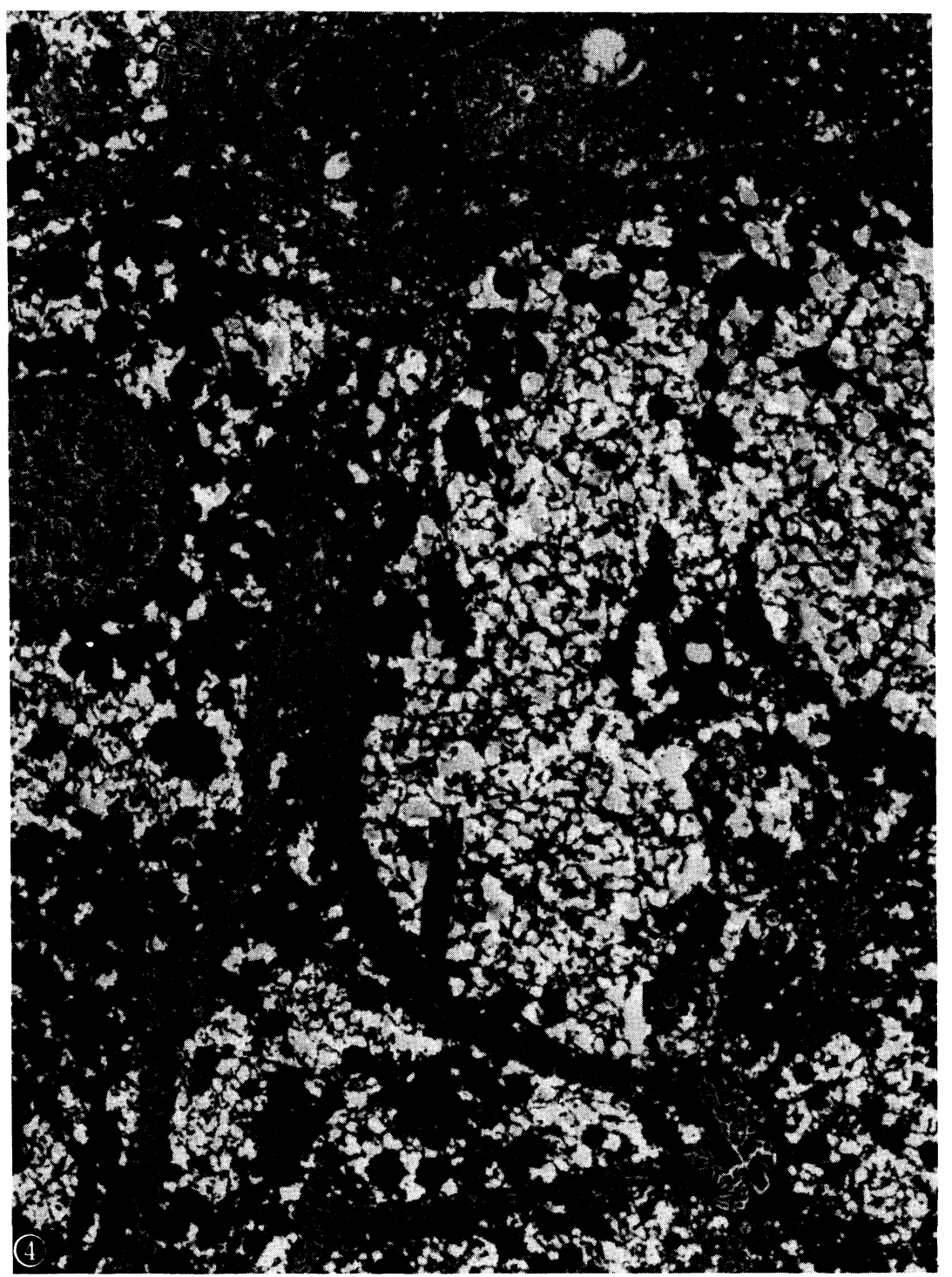

Fig. 4. Low magnification overview of epon-embedded section. Hepatocyte has pale cytoplasm with electron-dense reticular meshwork. Mitochondria (M) are numerous but endoplasmic reticulum (ER) is scanty. Membranes of cytoplasmic organelles and plasma membrane become indistinct. Intercellular space between hepatocytes appeared widened. $\times 4,300$ 


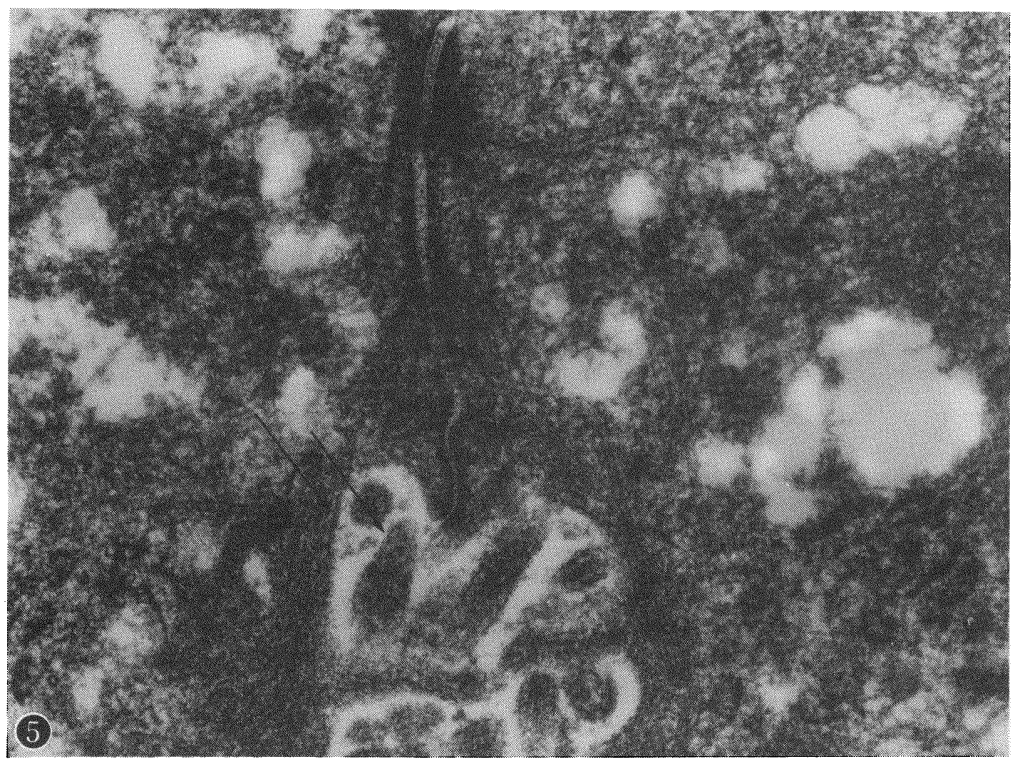

Fig. 5. Electron micrograph of ultrathin section illustrating the area around bile canaliculus. Triple-layered structure (arrows), though not distinct, can be observed. Fine thread-like structure, thicker filaments, and microtubules are present in the pericanalicular ectoplasm and in the cytoplasm. At the desmosome, granular meterial is present in the intercellular space. $\times 37,000$

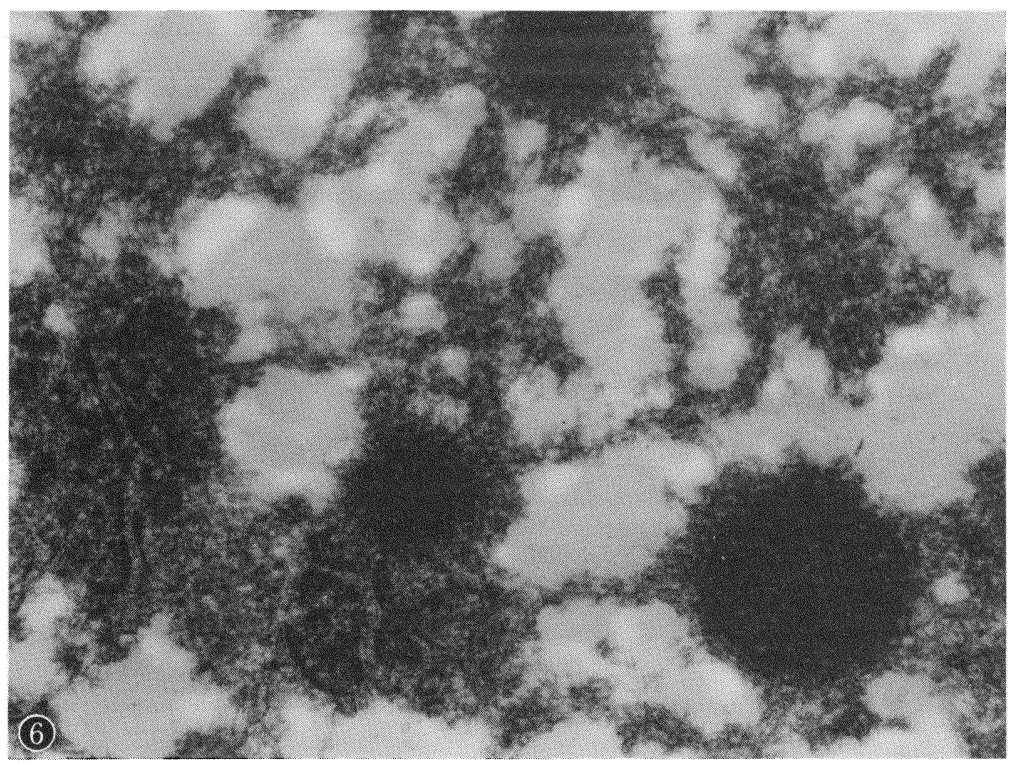

Fig. 6. Electron micrograph of the same section showing hepatocyte cytopplasm. Fibrillar substance coats the cytoplasmic organelles and connects them. We can observe the rough endoplasmic reticulum but the cisternae are not clearly seen. $\times 37,000$ 
are intact, and fat-storing cell and collagen fibers in the space of Disse are correctly located. In detailed structure of bile canaliculus, the pericanalicular network consisted of condensed fine filaments that were relatively straight (Fig. 2). Tight junction and desmosome could also be observed, but identification of the types of filament was impossible because too many filaments were densely superimposed upon each other.

In ultrathin sections from tissues treated in the same way and epoxyembedded, almost normal structure of liver could be seen except for the translucent areas in the cytoplasm that must be produced by the washout of cytosolic proteins (Fig. 3). At higher magnification near the bile canaliculus, almost intact morphology of bile canaliculus and junctional complexes as well as MFs, MTs, IFs and filamentous fuzzy materials (Ellisman and Porter 1980) could be observed (Fig. 4). MFs, MTs and filamentous fuzzy materials were mainly located around the bile canaliculus, and IFs were near the desmosome. Endoplasmic reticulum was not dilated, and filamentous fuzzy materials coated microbodies and radiated from them to connect with other cytoplasmic components (Fig. 5). This structure was thought to be equivalent to the cytoplasmic lattice seen in PEG-sections.

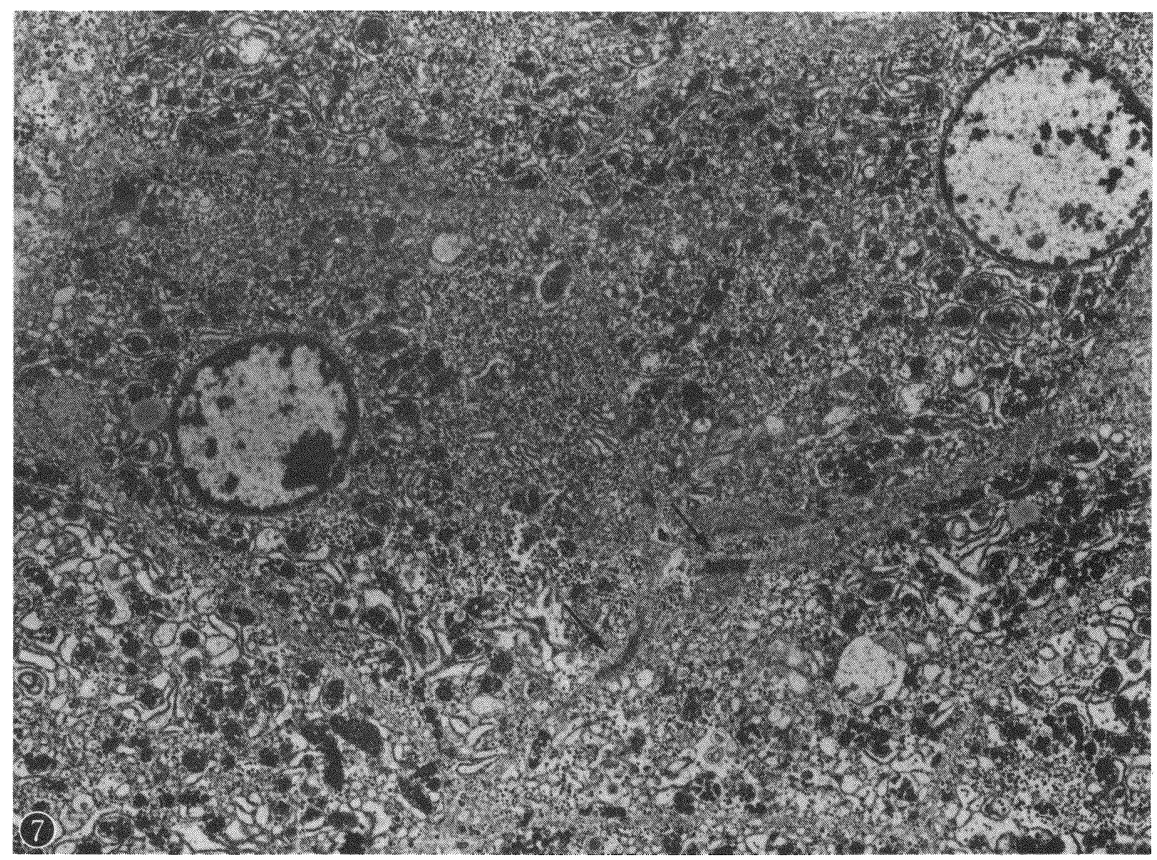

Figs. 7-14., except Fig. 10. ; Electron micrographs from sections processed as (B).

Fig. 7. Low magnification overview of epon-embedded section. Note the electron-dense reticular meshwork in the cytoplasm shown in Fig. 4 is absent. Intracytoplasmic organelles are markedly destroyed and plasma membrane is not distinct, but intercellular junctional complexes (arrows) look intact. $\times 3,500$ 


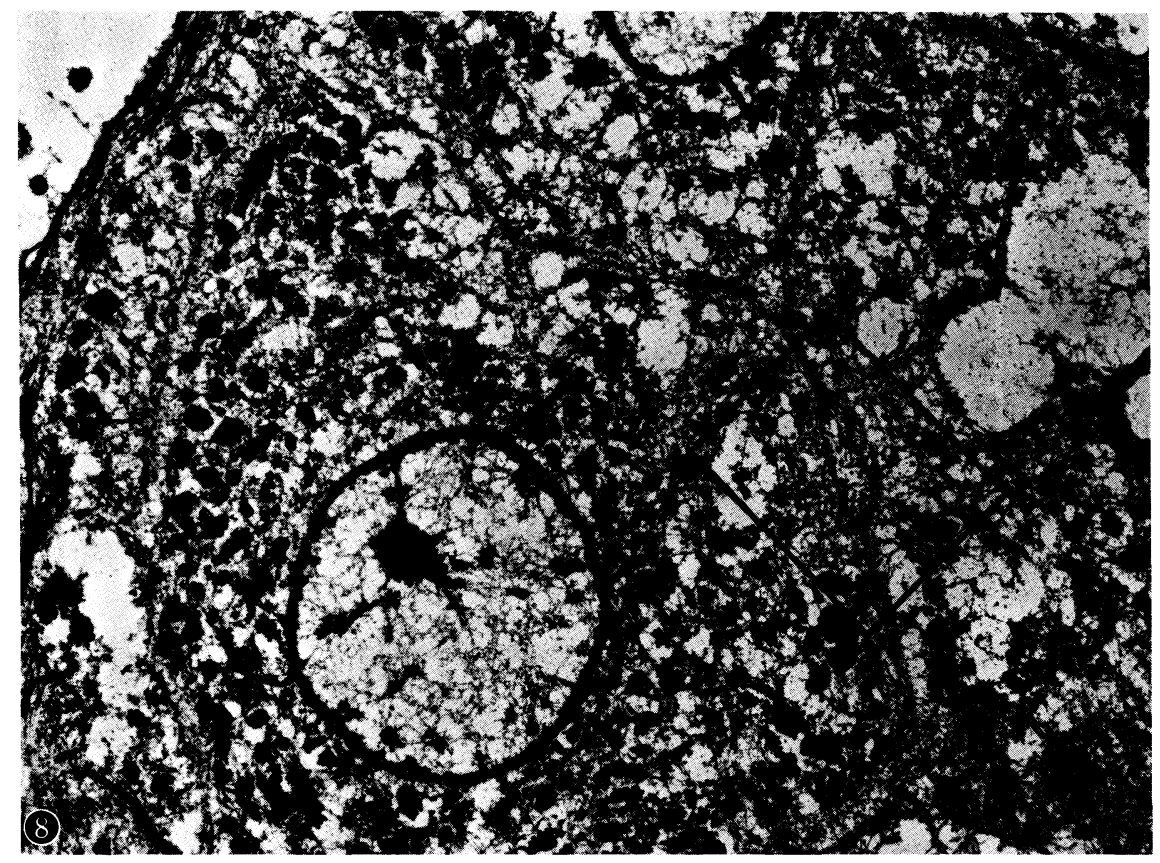

Fig. 8. Low magnification overview of PEG section. Fine filaments appear to distributed throughout the cytoplasm, but concentrated at the periphery of hepatocytes. Cytoplasmic organelles are markedly electron opaque because of thick section. $\times 3,200$

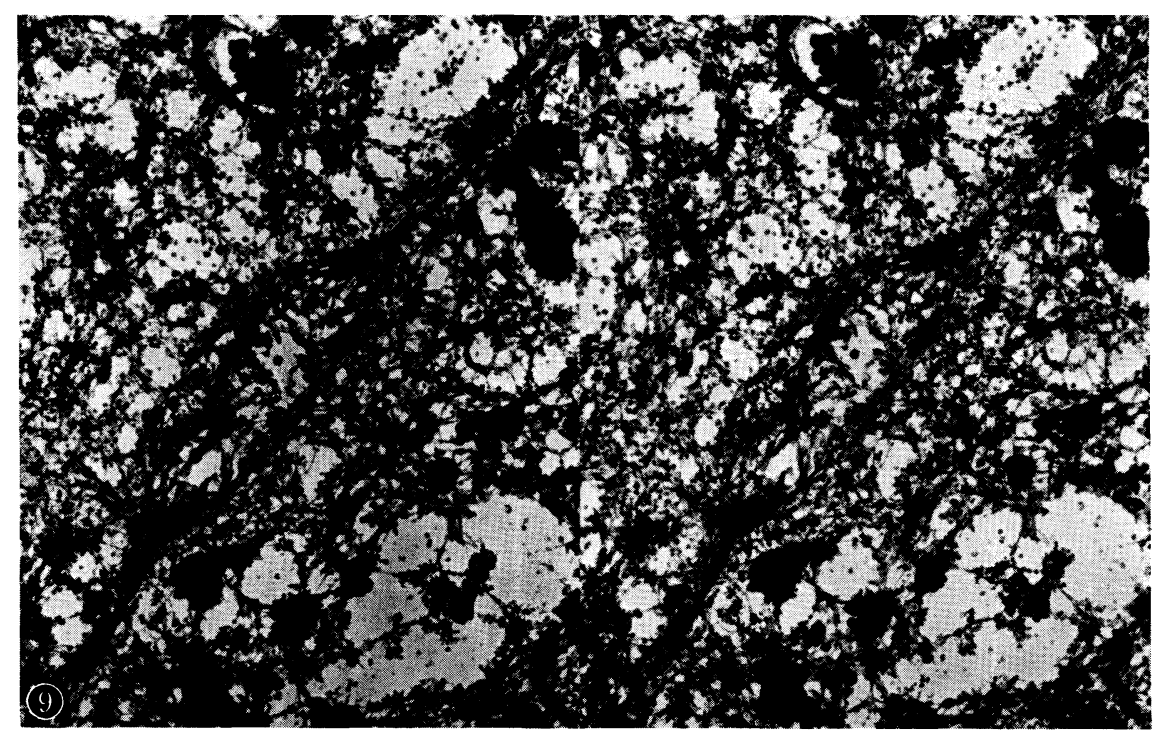

Fig. 9. Stereo pair micrographs of bile canaliculus encircled in Fig. 8. Note filaments, thought to be MFs, insert into each of the canalicular microvilli. Around the canalicular lumen, many filaments, though cannot be identified their types, encircled it and are attached to the junctional complex (arrows). $\times 8,600$ 
Cytoplasmic structure treated with procedure B (saponin and Triton $X-100$ )

At the peripheral part of the specimens hepatocytes had no MFs or cytoplasmic organelles (not shown here), and at the central part, as we can see in Fig. 9, there were fine filamentous structures composed of MFs, MTs and IFs; the presence of MFs and IFs in this area was also confirmed by immunofluorescence technique (not shown here). Fig. 6 shows the morphology of hepatocytes in PEG section. Comparing this with the micrograph of Fig. 1, hepatocytes appear to have much more disrupted organelles, nuclear chromatin, polysomes and filamentous structures. The bile canaliculus in Fig. 6 was further magnified in Fig. 7, where MFs were shown inserted into the microvilli and IFs located mainly near the junctional complexes and in the cytoplasm. Around the bile canaliculus, filament plexus, though the type of filaments could not be identified, encircled the canalicular lumen, and appeared to continue to the junctional
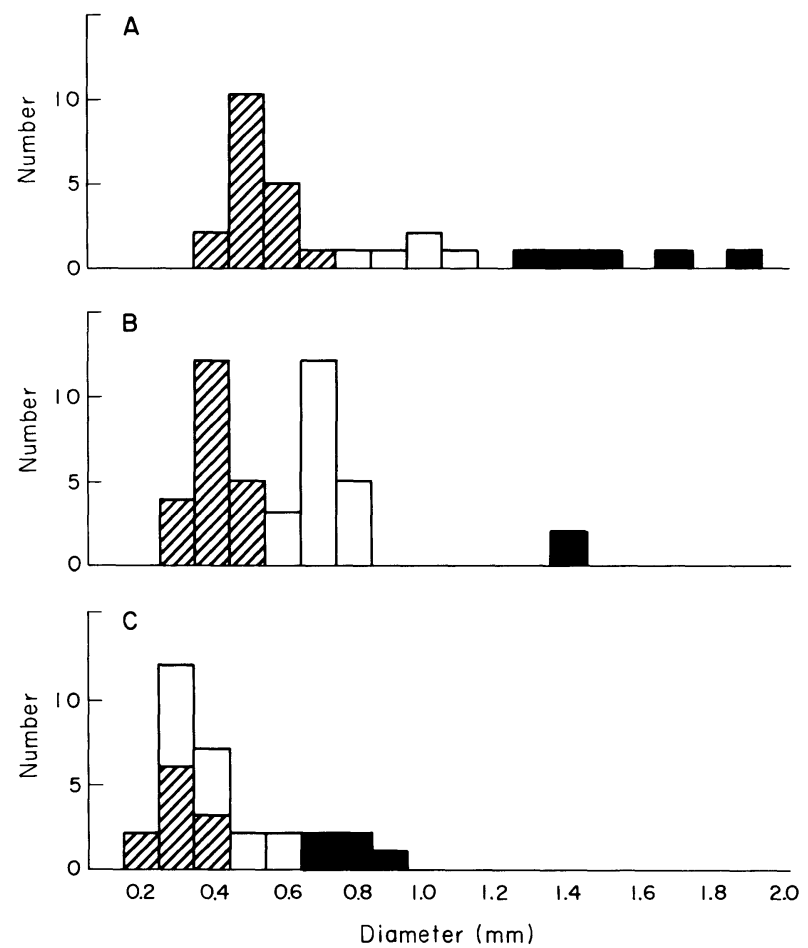

Fig. 10. Histograms showing the diameter distribution of MFs, MTs and IFs. Measurements were done from printed electron micrographs enalrged to 90,000 (A and $\mathrm{B}$ ) and to $30,000(\mathrm{C})$.

A : From epon-embedded section processed as (A).

B : From epon-embedded section processed as (B).

$\mathrm{C}$ : From PEG section processed as (B).

MFs VIII IFs $\square$ MTs 
complexes. MTs could occasionally be seen near the mitochondria. But the procedure of tissue preparation disrupted the organelles so severely that their structure was hardly discernible. Electron-dense lobulated material could be interpreted as the matrices of mitochondria (Fig. 7).

Fig. 8 shows how the three types of filaments are distributed in reference to their diameter, for procedures A and B. In PEG-sections treated with procedure A, diameters could not be measured because numerous filaments were superimposed to each other. In PEG-sections treated with procedure B, diameter of MFs was measured at the places of their insertion in the microvilli of canaliculus and that of IFs at the desmosome. Diameters of filaments in PEG-sections treated with procedure B were larger than those in epoxy-embedded sections, and the diameters of MFs and IFs were partially overlapping. So it is difficult in PEG-sections to discriminate MFs and IFs by their diameters. In ultrathin section, the diameters of filaments of hepatocytes treated with procedure B were smaller than those with procedure A. The results obtained in the latter state were almost the same as reported by others (Ishikawa et al. 1968, 1969; French and Davis 1975; Bershadsky et al. 1978; Yokota and Fahimi 1979; Fiskum et al. 1980), and clearly differentiated the types of filaments with their diameters.

From the observations in ultrathin sections, it was revealed that specimens

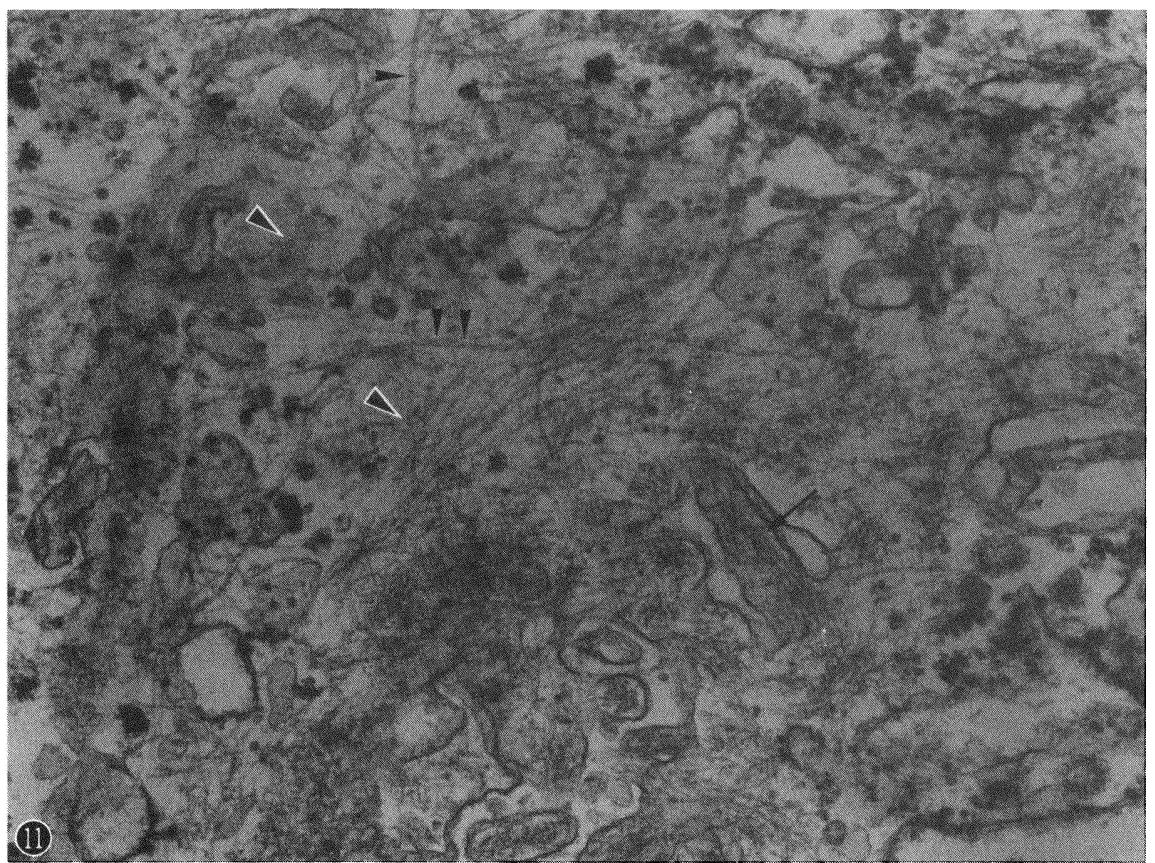

Fig. 11. Electron micrograph around the bile canaliculus. The disappearance of cytoplasmic matrix makes it clear to discern IFs $(\bowtie)$, MTs $(-)$ and MFs $(\rightarrow)$. Trilamellar structure of membrane is lost, but canalicular membrane is partially preserved. $\times 42,000$ 
treated with procedure B contained MFs, MTs and IFs (Fig. 9). IFs and MTs looked rich near the nucleus (Fig. 10). Around the bile canaliculus, each microvillus contained a bundle of actin filaments, and at the basal end of each bundle there was a fine plexus where the bundles terminated (Fig. 11). Some filaments of this plexus looked thicker in diameter than core microfilaments of microvilli and fuzzy in character. The plexus thus connected the actin bundles of microvilli, encircled the bile canaliculus (Fig. 11) and continued to its zonula adherens (Fig. 12). Outside the plexus, there were relatively small number of IFs

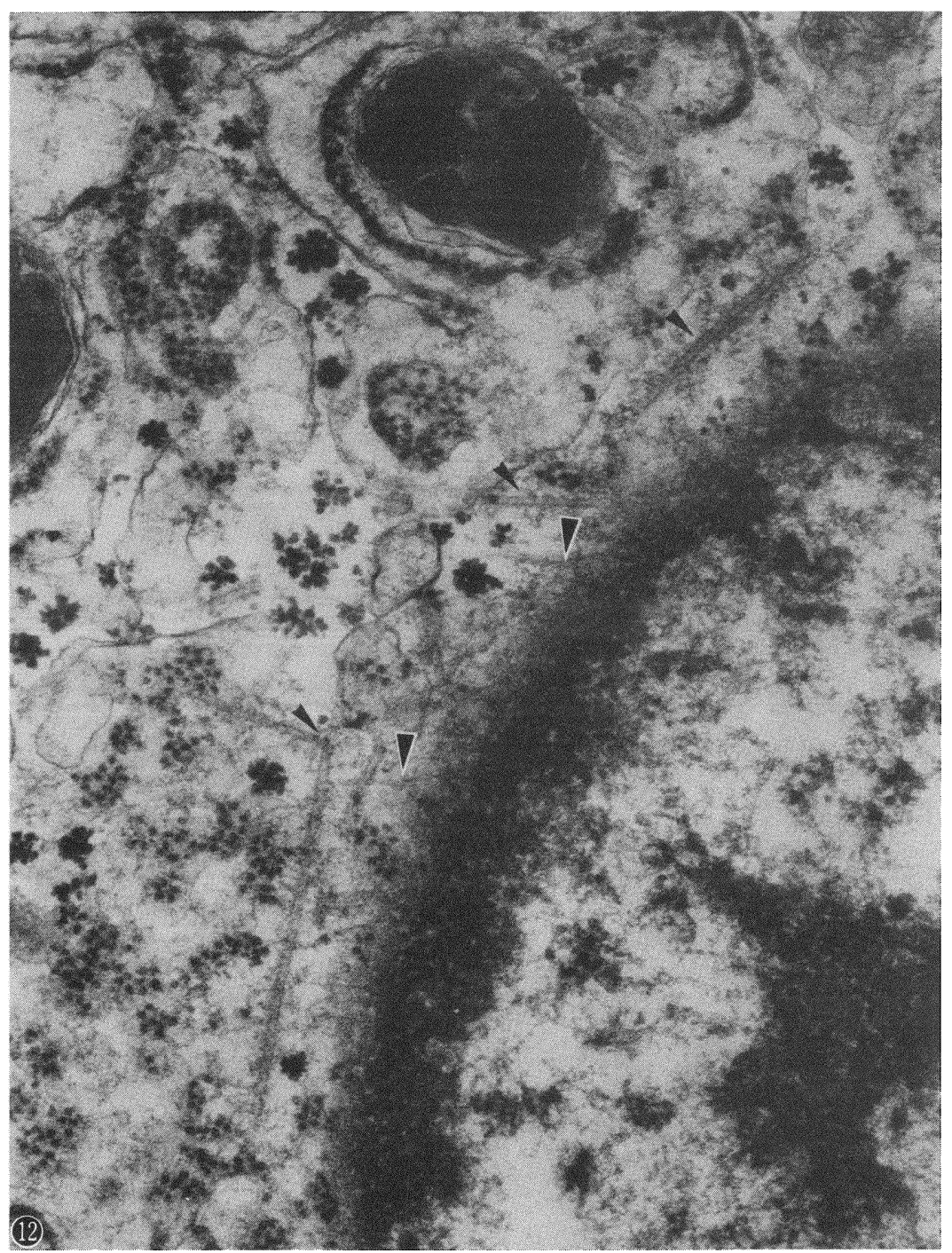

Fig. 12. Electron micrograph around the nucleus. IFs $(\bowtie)$ are rich around the nucleus. It looks as if they radiate perpendicularly from the nuclear surface. Also we can observe MTs $(\longrightarrow$ ) running along the nucleus. $\times 50,000$ 


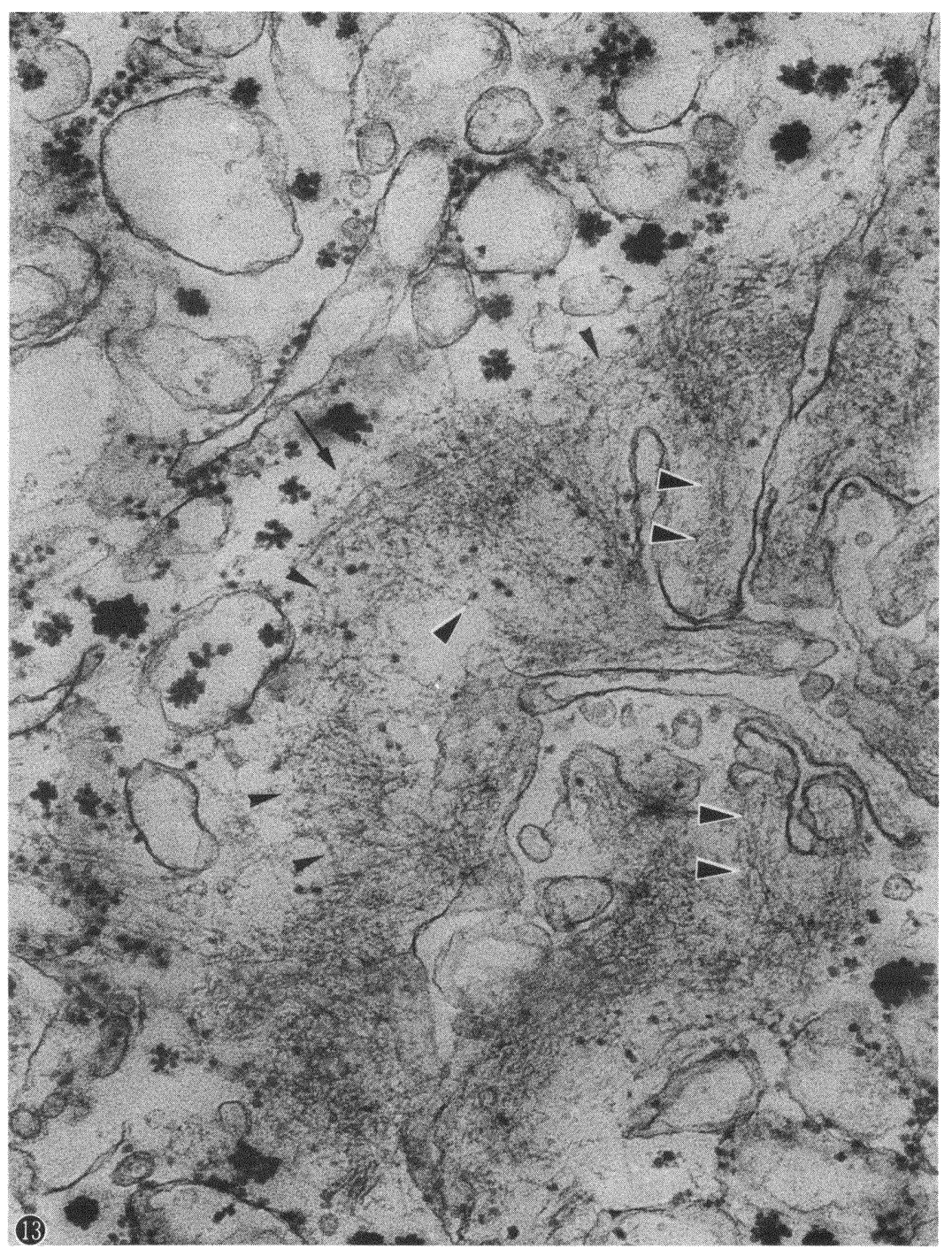

Fig. 13. Electron micrograph around the bile canaliculus. The core microfilaments in the microvilli $\leftrightarrow$ form bundles and terminate within the filament plexus $(-)$. The filaments of this plexus appear a little thicker than the core filaments. In and around the plexus, some IFs can be observed $(\rightarrow) . \quad \times 51,000$

that continued to the desmosome (Fig. 12).

\section{Discussion}

In hepatocytes, it has been well known that a filamentous system is present throughout the cytoplasm (Bieva 1964; Jahn 1980a, b), with MFs strongly condensed at the periphery of the cytoplasm, particularly around the bile canaliculi (Oda et al. 1974; Horoboroco et al. 1975). But their nature and 


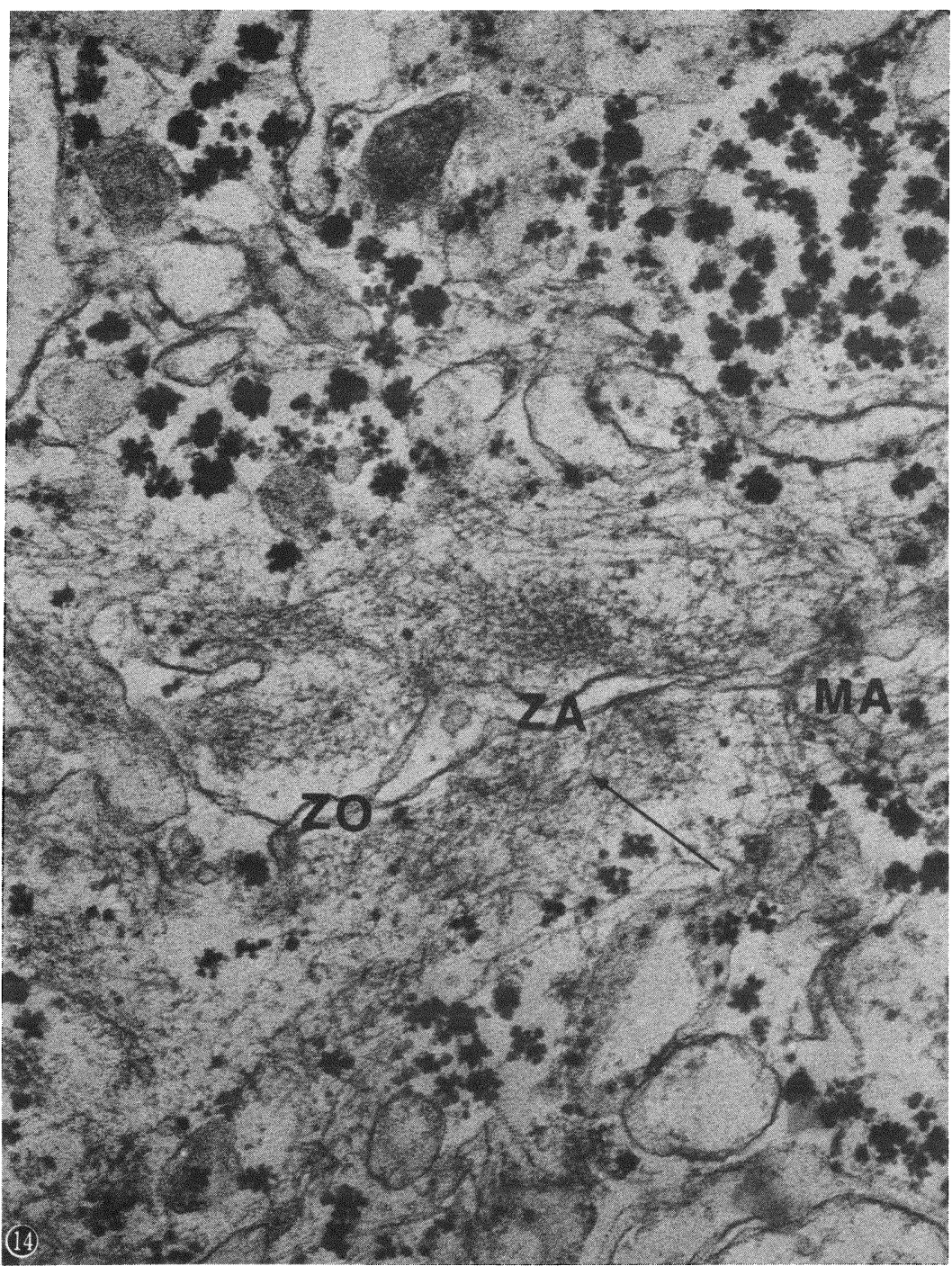

Fig. 14. Electron micrograph showing the area of junctional complex of bile canaliculus. Three components of junctional complex, i.e., tight junction (ZO), zonula adherens (ZA) and desmosome (MA), can be seen. Thin, microfilaments are attached to the ZA and $\mathrm{ZO}$, whereas IFs are attached to MA, and only a minor number of IFs was attached to $\mathrm{ZA}$ (arrows). $\times 66,800$

distribution remain to be elucidated.

Recently Wolosewick and Porter (1976, 1979) and Ellisman and Porter (1980) showed the three-dimensional lattice structure of cytoplasm, and since then the knowledge about the filamentous structure in the cytoplasm has progressed rapidly. In order to observe the cytoskeletal system of hepatocytes threedimensionally, we previously applied PEG-embedding method, first described by 
Wolosewick (1980), to rat hepatocytes and noticed in an observation at $200 \mathrm{kV}$ accelerating voltage that it was necessary to extract away cytosolic soluble proteins with detergent solution, even though the cytoskeleton of whole cultured hepatocyte (Ishii et al. 1983) or nerve cell (Nagele et al. 1983) could be demonstrated without this treatment at the same accelerating voltage, possibly because the latter contained few cytosolic proteins. In a preliminary study using rat hepatocyte, we removed the soluble proteins with Triton X-100 and could demonstrate the cytoskeleton clearly, but membranous structure was partially or completely destroyed, leaving the relationship between plasma membrane and cytoskeleton quite ambiguous (Ishii et al. 1984). So in this study, we used two fixation schedules; procedure $\mathrm{A}$ was to preserve the plasma membrane and procedure $\mathrm{B}$ to denude the cytoskeleton by destroying membranous structures.

When treated with procedure A, as shown by Maupin and Pollard (1983) on HeLa cells, hepatocytes showed pale cytoplasm with organelles, nucleus and plasma membrane kept almost intact. Fine lattice structure other than MFs, MTs and IFs could be observed with satisfactory contrast in unstained PEG-sections, possibly because of absent embedding medium (Wolosewick 1980). This lattice structure extended throughout the cytoplasm and was condensed in the submembranous cytoplasm, particularly around bile canaliculi. The distribution and electron microscopic nature of this structure seemed to be the same as Bieva (1964) showed in ultrathin section, and the three-dimensional organization of this structure was the same as shown by Wolosewick and Porter $(1976,1979)$ and Ellisman and Porter (1980) who, in chick embryo fibroblasts and rat nerve cells, termed this fibllar system "microtrabecular lattice". As demonstrated by Wolosewick and Porter (1979) in cultured cells, microtrabecular lattice appeared to stick on the cytoplasmic organelles and plasma membranes, and connect with each other. Microtrabecular lattice has been reported to comprise different kinds of proteins including 200-k daltone proteins (Hirokawa 1982), MAPs (Griffith and Pollard 1978), myosin (Horoboroco et al. 1975; Hirokawa et al. 1982), macrophage actin-binding protein (Heuser and Kirschner 1980; Hartwig and Stossel 1981; Niederman et al. 1983), $\alpha$-actinin (Jockusch and Isenberg 1981), vinculin (Jockusch and Isenberg 1981) and so on (Weeds 1982). These proteins are thought to regulate cell architecture and motility (Weeds 1982). But the nature of lattice structure in human hepatocytes is as yet unknown, and biochemical and immunohistochemical analyses are necessary to elucidate the nature and the role of the lattice structure of hepatocytes observed here.

The washout of cytoplasm with procedure A was not enough for the observation of the other components of filaments, i.e., MFs, MTs and IFs because too many lattice filaments hindered their discrimination.

On the other hand, when treated with procedure $\mathrm{B}$, residual cytoskeletons resistant to saponin and Triton X-100 were only IFs at the peripheral area; at the central area of the block MFs, MTs and IFs were also found to remain because of 
incomplete and/or delayed washout. So in the central area, where the major lattice structure and other cytoplasmic organelles were removed, MFs, MTs and IFs were visualized more clearly than in the specimens treated with procedure A.

In the central area of PEG-sections, we could confirm the meshwork distribution of filaments with pericanalicular condensation. But whether indivividual filaments were MFs or IFs could not be identified, not only because in PEGsections most filaments appeared thicker than reported by others, but also because the range of diameter was overlapping among different types of filaments. Strict discrimination of these types requires electron microscopy on usual ultrathin section.

Of special interest was the distribution of filaments around the bile canaliculi. It is well known that this area, termed pericanalicular ectoplasm, is rich in MFs (Bieva 1964). But their detailed structure is not known. In this study, we showed that MFs and IFs were distributed in an orderly fashion. In the pericanalicular area, each microvillus of canaliculus contains a core of filament bundle with associated filament plexus at its base, and the plexus was continued to zonula adherens. The filaments of the plexus appear more thick and fuzzy, compared with those of the core bundle in microvilli. At present, it is not clear whether these filaments are composed of actin filaments. IFs were located in and around the plexus and continued to the desmosome and zonula adherens. These structures are surprisingly similar to those found in the brush border of intestinal epithelia (Hull and Staehelin 1979).

As to the brush border of intestinal epithelia, many reports demonstrated that an active contraction could occur at the terminal web region (Burgess 1982; Mooseker 1976). Our study of human hepatocytes showed that the filament plexus around bile canaliculi appeared quite similar to the intestinal terminal web not only in appearance but in its attachment to the zonula adherens. From this morphological analogy in the filament distribution, it is interesting to speculate that bile canaliculi are capable of active contraction like an intestinal epithelium. Recently, Oshio and Phillips (1981), and Phillips et al. (1982, 1983) showed that cultured rat hepatocytes really had a forceful contraction ability. As yet, there has been no clear evidence that bile canaliculi in human do contract in vivo, and further study must be performed to substantiate this speculation.

\section{References}

1) Bershadsky, A.D., Gelfand, V.I., Suitkina, T.M. \& Tint, I.S. (1978) Microtubules in mouse embryo fibroblasts extracted with Triton X-100. Cell Biol. int. Rep., 2, 425432.

2) Bieva, C.G. (1964) Studies on cholestasis. A re-evaluation of the fine structure of normal human bile canaliculi. Lab. Invest., 13, 840-864.

3) Burgess, D.R. (1982) Reactivation of intestinal epithelial cell brush border motility: ATP-dependent contraction via a terminal web contractile ring. J. Cell Biol., 95, 853-863. 
4) Ellisman, M.H. \& Porter, K.R. (1980) Microtrabecular structure of the axoplasmic matrix: visualization of cross-linking structures and their distribution. $J$. Cell Biol., 87, 464-479.

5) Fiskum, G., Craig, S.W., Decker, G.L. \& Lehninger, A.L. (1980) The cytoskeleton of digitonin-treated rat hepatocytes. Proc. nat. Acad. Sci. USA, 77, 3430-3434.

6) French, S.W. \& Davis, P.L. (1975) Ultrastructural localization of actin-like filaments in rat hepatocytes. Gastroenterology, 68, 765-774.

7) Griffith, L.M. \& Pollard, T.D. (1978) Evidence of actin-filament-microtubule interaction mediated by microtubule-associated proteins. J. Cell Biol., 78, 958-965.

8) Hartwig, J.H. \& Stossel, T.P. (1981) Structure of macrophage actin-binding protein molecules in solution and interacting with actin filaments. $J$. mol. Biol., 145, 563-581.

9) Heuser, J.E. \& Kirschner, M.W. (1980) Filament organization revealed in platinum replicas of freeze-dried cytoskeletons. J. Cell Biol., 86, 212-234.

10) Hirokawa, N. (1982) Cross-linker system between neurofilaments, microtubules, and membranous organelles in frog axon revealed by the quick-freeze, deep etching method. J. Cell Biol., 94, 129-142.

11) Hirokawa, N., Tilney, L.G., Fujiwara, K. \& Heuser, J.E. (1982) Organization of actin, myosin, and intermediate filaments in the brush border of intestinal epithelial cells. J. Cell Biol., 94, 425-443.

12) Horoboroco, E.J., Trencher, E.J., Dorling, J. \& Webb, J. (1975) Demonstration of smooth muscle contractile protein antigens in liver and epithelial cells. Ann. N.Y. Acad. Sci., 154, 489-504.

13) Hull, B.E. \& Staehelin, L.A. (1979) The terminal web. A reevaluation of its structure and function. J. Cell Biol., 81, 67-82.

14) Ishii, M., Shimizu, A., Suzuki, H. \& Otsuki, M. (1983) Visualization of cytoskeletons and cell membranes by a whole cell mount method-Electron microscopic observations of cultured cells after treatment of cytochalasin B, colchicine and dexamethasone. $J$. clin. Electron Microsc., 16, 1-10.

15) Ishii, M., Suzuki, H., Ohta, S., Otsuki, M. \& Goto, Y. (1984) Morphologic study of cytoskeletal systems of mouse hepatocytes using polyethylene glycol-embedding method. Tohoku J. exp. Med., 142, 299-311.

16) Ishikawa, H., Bischoff, R. \& Holtzer, H. (1968) Mitosis and intermediate-sized filaments in developing skeletal muscle. J. Cell Biol., 38, 538-555.

17) Ishikawa, H., Bischoff, R. \& Holtzer, H. (1969) Formation of arrowhead complaxes with heavy meromyosin in a variety of cell types. $J$. Cell Biol., 43, 312-328.

18) Jahn, W. (1980a) Visualization of a filament network in cryo sections of liver tissue. Europ. J. Cell Biol., 20, 301-304.

19) Jahn, W. (1980b) The cytoskeleton of rat liver parenchymal cells. Naturwissenschaften, 67, 568 .

20) Jockusch, B.M. \& Isenberg, G. (1981) Interaction of $\alpha$-actinin and vinculin with actin: opposite effects on filaments network formation. Proc. nat. Acad. Sci. USA, 78, 3005-3009.

21) Maupin, P. \& Pollard, T.D. (1983) Improved preservation and staining of HeLa cell actin filaments, clathrin-coated membranes, and other cytoplasmic structures by tannic acid-glutaraldehyde-saponin fixation. J.Cell Biol., 96, 51-62.

22) Mooseker, M.S. (1976) Brush border motility. Microvillar contraction in Tritontreated brush borders isolated from intestinal epithelium. J. Cell Biol., 71, 417-433.

23) Nagele, R.G., Roisen, F.J. \& Lee, H. (1983) A method for studying the threedimensional organization of cytoskeletal elements of cells: improvements in the polyethylene glycol technique. J. Microsc., 129, 179-184.

24) Niederman, R., Amrein, P.C. \& Hartwig, J. (1983) Three-dimensional structure of actin filaments and of an actin gel made with actin-binding protein. J. Cell Biol., 96, $1400-1413$. 
25) Oda, M., Price, V.M., Fischer, M.M. \& Phillips, M.J. (1974) Ultrastructure of bile canaliculi, with special reference to the surface coat and the pericanalicular web.Lab. Invest., 31, 314-323.

26) Oshio, C. \& Phillips, M.J. (1981) Contractility of bile canaliculi : implications for liver function. Science, 212, 1041-1042.

27) Phillips, M.J., Oshio, C., Miyairi, M., Katz, H. \& Smith, C.R. (1982) A study of bile canalicular contractions in isolated hepatocytes. Hepatology, 2, 763-768.

28) Phidllips, M.J., Oshio, C., Miyairi, M. \& Smith, C.R. (1983) Intrahepatic cholestasis as a canalicular motility disorder. Evidence using cytochalasin. Lab. Invest., 48, $205-211$.

29) Schliwa, M. \& van Blerkom, J. (1981) Structural interaction of cytoskeletal components. J. Cell Biol., 90, 222-235.

30) Weeds, A. (1982) Actin-binding proteins-Regulators of cell architecture and motolity. Nature, 296, 811-816.

31) Wolosewick, J.J. (1980) The application of polyethylene glycol (PEG) to electron microscopy. J. Cell Biol., 86, 675-681.

32) Wolosewick, J.J. \& Porter, K.R. (1976) Stereo high-voltage electron microscopy of whole cells of the human diploid line, W1-38. Amer. J. Anat., 147, 303-324.

33) Wolosewick, J.J. \& Porter, K.R. (1979) Microtrabecular lattice of the cytoplasmic ground substance. Artifact or reality. J. Cell Biol., 82, 114-139.

34) Yokota, S. \& Fahimi, H.D. (1979) Filament bundles of prekeratin types in hepatocytes: revealed by detergent extraction after glutaraldehyde fixation. Biol. cell., 34, 119-126. 